\title{
Determinantes da judicialização da saúde: uma análise bibliográfica
}

\author{
Determinants of health judicialization: a bibliographical analysis
}

Determinantes de la judicialización de la salud: un análisis bibliográfico

André Luís Bonifácio de Carvalho ${ }^{1}$

Andrey Maia Silva Diniz²

Bianca Nóbrega de Medeiros Batista ${ }^{3}$

Daniella de Souza Barbosa ${ }^{4}$

Edjavane da Rocha Rodrigues de Andrade Silva ${ }^{5}$

Otávio Augusto Nasser Santos 6

Raquel Veloso do Nascimento ${ }^{7}$

\section{Resumo}

Objetivo: a judicialização da saúde é um fenômeno multifacetado e requer a construção de caminhos que possibilitem a análise de fatores que, isolada ou conjuntamente, influenciam em sua constituição; assim, torna-se necessário identificar quais são os fatores apontados pela literatura científica nacional como causas da judicialização no campo da saúde. Metodologia: foi feita uma revisão integrativa de literatura, fundamentada no estudo exploratório-descritivo e bibliográfico de 101 publicações contidas na base de dados da Coordenação de Aperfeiçoamento de Pessoal de Nível Superior, usando cinco descritores compatíveis com a pesquisa, a saber: judicialização e saúde e Brasil; judicialização da saúde e Direito; judicialização e saúde e causas; judicialização e saúde e consequências; e judicialização e direito à saúde. Resultados: dos 101 artigos catalogados, 30 foram selecionados; tal amostra foi organizada em três categorias, a partir de sua temática: 14 artigos discutiam a judicialização da assistência farmacêutica; 10 artigos abordavam a judicialização do direito à saúde; e 6 artigos debatiam sobre a judicialização com ênfase nas práticas de gestão. Conclusão: ainda é incipiente a produção intelectual brasileira para a identificação e sistematização dos determinantes da judicialização. Contudo, foi possível identificar caminhos que permitiram uma leitura preliminar sobre a temática, que foram

\footnotetext{
${ }^{1}$ Doutor em Ciências da Saúde, Universidade de Brasília (UnB), Brasília, Distrito Federal, Brasil; professor adjunto I, Departamento de Promoção da Saúde, Centro de Ciências Médicas, Universidade Federal da Paraíba, João Pessoa, Paraíba, Brasil. https://orcid.org/0000-0003-0328-6588. E-mail: andrelbc4@gmail.com

2 Graduando em Medicina, Universidade Federal da Paraíba, João Pessoa, Paraíba, Brasil. https://orcid.org/0000-00025572-7018. E-mail: andreymaiasd@gmail.com

${ }^{3}$ Graduanda em Fisioterapia, Universidade Federal da Paraíba, João Pessoa, Paraíba, Brasil. https://orcid.org/0000-00017865-1311. E-mail: biancanobregamed@gmail.com

4 Doutora em Educação, Universidade Federal da Paraíba, João Pessoa, Paraíba, Brasil; professora adjunta I, Departamento de Promoção da Saúde, Centro de Ciências Médicas, Universidade Federal da Paraíba, João Pessoa, Paraíba, Brasil. https://orcid.org/0000-0003-3533-146X. E-mail: daniella.77.fcm@gmail.com

${ }^{5}$ Mestranda em Saúde Coletiva, Universidade Federal da Paraíba, João Pessoa, Paraíba, Brasil. https://orcid.org/00000002-7854-5751. E-mail: edjavanerocha@hotmail.com

${ }^{6}$ Graduando em Medicina, Universidade Federal da Paraíba, João Pessoa, Paraíba, Brasil. https://orcid.org/0000-00015489-0545. E-mail: oansg28@gmail.com

${ }^{7}$ Graduanda em Medicina, Universidade Federal da Paraíba, João Pessoa, Paraíba, Brasil. https://orcid.org/0000-00024814-7488. E-mail: raquelveloso98@hotmail.com
} 
divididos em três categorias: judicialização da assistência farmacêutica; judicialização do direito à saúde; e judicialização e práticas de gestão.

\title{
Palavras-chave
}

Judicialização da assistência farmacêutica. Judicialização do direito à saúde. Judicialização da saúde pública. Judicialização da saúde.

\begin{abstract}
Objective: health judicialization is a multifaceted phenomenon; thus, it is necessary to identify what are the factors that cause this phenomenon in Brazilian scientific literature. Methods: it was conducted an integrative literature review, based on an exploratorydescriptive and bibliographic study of 101 publications available in Capes database. The search used 5 descriptors: judicialization and health and Brazil; health judicialization and law; judicialization and health and causes; judicialization and health and consequences; and judicialization and the right to health. Results: of a corpus of 101 articles, the authors selected 30, which were organized into three categories based on its theme: 14 articles discussed the judicialization of pharmaceutical assistance; 10 articles addressed the judicialization of the right to health; and 6 articles discussed judicialization with emphasis on management practices. Conclusion: Brazilian intellectual production is still incipient to identify and systematize the determinants of health judicialization. However, it was possible to have a preliminary understanding of the main themes, which were divided into three categories: the judicialization of pharmaceutical assistance, the judicialization of the right to health and the judicialization and management practices.
\end{abstract}

\section{Keywords}

Judicialization of pharmaceutical assistance. Judicialization of the right to health. Judicialization of public health. Health judicialization.

\section{Resumen}

Objetivo: la judicialización de la salud es un fenómeno multifacético y requiere la construcción de caminos que permitan analizar los factores que, individualmente o en conjunto, inciden en su constitución; por tanto, se hace necesario identificar cuáles son los factores señalados por la literatura científica nacional como causas de judicialización en el campo de la salud. Metodología: se realizó una revisión integradora de la literatura, a partir de un estudio exploratorio-descriptivo y bibliográfico de 101 publicaciones contenidas en la base de datos de la Coordinación para el Perfeccionamiento del Personal de Educación Superior, utilizando cinco descriptores compatibles con la investigación, a saber: judicialización y salud. y Brasil; judicialización de la salud y el derecho; judicialización y salud y causas; judicialización y salud y consecuencias; y judicialización y derecho a la salud. Resultados: de los 101 artículos catalogados, se seleccionaron 30; esta muestra se organizó en tres categorías, en función de su temática: 14 artículos discutieron la judicialización de la asistencia farmacéutica; 10 artículos abordaron la judicialización del derecho a la salud; y 06 artículos discutieron la judicialización con énfasis en las prácticas de gestión. Conclusión: la producción intelectual brasileña es aún incipiente para la identificación y sistematización de los determinantes de la judicialización. Sin embargo, fue posible identificar caminos que permitieron una lectura preliminar sobre el tema, que se dividió en tres categorías: la primera, judicialización de la asistencia farmacéutica, seguida de la judicialización del derecho a la salud y finalmente, judicialización y prácticas de gestión.

\section{Palabras clave}

Judicialización de la asistencia farmacéutica. Judicialización del derecho a la salud. Judicialización de la salud pública. Judicialización de la salud. 


\section{Introdução}

A Constituição de 1988, em seu art. 196, estabeleceu a saúde como direito de todos e dever do Estado, definindo o acesso às ações e aos serviços de saúde como uma condição inerente à cidadania no Brasil (1). Tal disposição constitucional não só alterou as relações entre o Estado e a Sociedade no âmbito do sistema de proteção social do país, como também mudou a dinâmica de relações entre os poderes públicos, conferindo novo status ao poder judiciário como potencial esfera garantidora do acesso e da utilização de tais serviços aos cidadãos. Desde então, a estratégia de recorrer à via jurídica para exigir a garantia do direito tem sido uma prática crescente em todos os estados brasileiros, tornando o fenômeno da judicialização da saúde um dos principais temas da agenda nacional da saúde (2).

Empregada, inicialmente, na primeira metade dos anos 1990, por portadores de HIV para exigir que a União garantisse o fornecimento de medicamentos antirretrovirais, seu uso se diversificou nas décadas seguintes, tornando-se uma porta de entrada específica e paralela às vias gerenciais do Sistema Único de Saúde (SUS), para o acesso a um amplo conjunto de bens e serviços no campo da saúde (2). A dimensão e a relevância que a judicialização da saúde tem assumido, nos últimos anos, podem ser percebidas tanto pela evolução de seus impactos financeiros quanto pelas iniciativas político-institucionais e técnicas desenvolvidas em âmbito nacional e regional para gerenciar sua evolução, como verificado pela consultoria jurídica da Advocacia Geral da União junto ao Ministério da Saúde, no período de 2007 a 2016, no qual houve um aumento de quase $5.000 \%$ nos gastos com judicialização: de $R \$ 26$ milhões (2007) para mais de $R \$ 1,325$ bilhão de reais (2016), só no âmbito da União $(3,4)$.

Em 2009, o Supremo Tribunal Federal (STF) convocou uma audiência pública nacional de ampla representação para debater as diferentes visões sobre a judicialização da saúde e apontar os caminhos. As principais estratégias estabelecidas para lidar com a judicialização da saúde, a partir desse debate, consistiram na instituição de espaços de diálogo entre os Poderes Judiciário e Executivo, no fortalecimento da regulação federal sobre o mercado nacional de bens e serviços em saúde, na definição de diretrizes para a incorporação de tecnologias em saúde no SUS e na instalação de instâncias de assessoramento técnico em saúde aos magistrados (5). A implementação do direito à saúde vem ganhando contornos peculiares, obrigando os operadores do direito em diversas instâncias de atuação a lidarem com temas vinculados ao Direito Sanitário e a sua correlação 
com a implantação das políticas públicas de saúde nos três níveis de governo. De maneira correlata, os gestores públicos de saúde são permanentemente provocados a lidarem com a garantia efetiva desse direito social, seja individual ou coletivamente, por meio de determinações oriundas do Poder Judiciário e que, por vezes, contradiz o que está preconizado na política pactuada da assistência à saúde, como também os processos que dizem respeito à logística do sistema de saúde (6).

Cabe destacar que a Constituição Federal de 1988 garante o direito a saúde como um direito social, mediante a elaboração de políticas sociais e econômicas por parte do Estado. Por sua vez, as políticas públicas destinam-se a racionalizar a prestação coletiva do Estado, baseando-se, para tanto, nas principais necessidades de saúde da população, buscando promover a tão aclamada justiça distributiva, inerente à própria natureza dos direitos sociais, representando assim a própria garantia desses direitos (7-10).

Uma das recentes evoluções jurídicas no SUS foi o Decreto no 7.508/2011, de 28 de junho de 2011, que regulamentou a Lei no 8.080, de 19 de setembro de 1990, dispondo sobre a organização o planejamento da saúde, a assistência à saúde e a articulação interfederativa e outras providências (7). Marques e Dallari (11) sustentam que as políticas públicas estabelecidas em matéria de assistência à saúde devem ser conhecidas pelo Poder Judiciário, ao garantir efetivamente o direito à saúde nos casos concretos que são submetidos à sua apreciação, pois, dessa maneira, seria possível conjugar os interesses individuais com os coletivos, formalizados mediante tais políticas.

Segundo Marques (6), se por um lado a crescente demanda judicial acerca do acesso a medicamentos, produtos para a saúde, cirurgias, leitos de UTI, dentre outras prestações positivas de saúde pelo Estado, representa um avanço em relação ao exercício efetivo da cidadania por parte da população brasileira, por outro, significa um ponto de tensão perante os elaboradores e executores da política no Brasil, pois passam a atender um número cada vez maior de ordens judiciais, que representam gastos públicos e ocasionam impactos significativos na gestão pública da saúde no País.

Segundo Pepe et al (3), a judicialização da saúde é um fenômeno multifacetado, que expõe limites e possibilidades institucionais estatais e instiga a produção de respostas efetivas pelos agentes públicos do setor saúde e do sistema de justiça. A intervenção judicial no âmbito da gestão do setor da saúde tem sido alvo de intenso debate e, recentemente, ganhou destaque no STF, com a realização de audiência pública que possibilitou a interlocução entre atores envolvidos. 
A esse novo papel exercido pelo Poder Judiciário na garantia de direitos individuais, tem sido atribuída a noção de judicialização (12). Com o aumento exponencial das ações e a impossibilidade de previsão orçamentária dos gastos por elas acarretados, os gestores do sistema de saúde, nos três âmbitos, tentam resolver de diversas maneiras os impasses criados. Um dos desafios é conhecer as principais demandas judiciais e promover políticas públicas que atendam às necessidades que, na judicialização, pode aparecer como individual, mas que se constitui um fenômeno de muitos e, por isso, torna-se uma demanda coletiva, indicando a necessidade de reorganização da oferta de políticas públicas, na tentativa de diminuir as demandas judiciais e as despesas cada vez mais imprevisíveis nos orçamentos estaduais. Qualificando a discussão, Fleury (13), destaca que a judicialização da saúde no Brasil foi vista até agora como uma interferência indevida sobre a capacidade de planejamento e ação do Executivo e como uma ameaça à ação dos gestores locais. A autora crê que essa fase está sendo superada e defende que a judicialização é, hoje, a maior aliada do SUS.

Conhecer esse fenômeno, que é multifacetado, requer a construção de caminhos que possibilitem a análise de fatores que, isolada ou conjuntamente, influem em sua constituição. O presente artigo busca problematizar a seguinte questão norteadora: quais são os fatores apontados pela literatura científica nacional como causas da judicialização no campo da saúde? Para tanto se faz necessário o aprofundamento do tema e a identificação dos elementos que o induzem, seja no âmbito da gestão, na conformação do perfil dos demandantes; na organização da agenda dos gestores e na dinâmica de implementação das políticas.

\section{Metodologia}

Foi realizada uma revisão integrativa de literatura, fundamentada no estudo exploratório-descritivo e bibliográfico. A partir da identificação, localização e compilação dos dados escritos em artigos de revistas especializadas (13), do acervo da Coordenação de Aperfeiçoamento de Pessoal de Nível Superior (Capes), foi feita uma coleta de dados realizada a partir de fontes secundárias -, por meio do cumprimento de seis etapas metodológicas para elaboração do corpus documental da presente pesquisa.

Tais etapas foram assim organizadas, em sua ordem cronológica: 1) escolha da questão norteadora ("quais os determinantes do processo de judicialização da saúde no Brasil?"); 2) busca no banco de dados online da Capes, a partir dos descritores selecionados 
(judicialização e saúde e Brasil; judicialização da saúde e Direito; judicialização e saúde e causas; judicialização e saúde e consequências; e judicialização e direito à saúde); 3) definição dos critérios de inclusão (artigos publicados na íntegra e em língua portuguesa a partir do ano de 2008; conter a temática de pelo menos um dos descritores listados acima; responder a pergunta norteadora) e de exclusão (ser artigo de revisão de literatura; ser tese, dissertação, trabalho monográfico ou de conclusão de curso); 4) avaliação e organização dos artigos incluídos nos resultados, a partir das seguintes categorias temáticas: judicialização da assistência farmacêutica; judicialização do direito à saúde; e judicialização da saúde pública); 5) discussão dos resultados à luz da literatura vigente com relação à temática trazida pela questão norteadora; e 6) apresentação da revisão integrativa (14).

A proposta sugerida para a identificação dos determinantes da judicialização da saúde no Brasil foi a questão norteadora da pesquisa. Combinados, por meio do operador de pesquisa (booleano) AND no banco de dados da Capes, foram encontrados, respectivamente, 34 estudos para os descritores 1; 28 estudos para o 2; 16 estudos para o 3; 9 estudos para o 4; e 14 estudos para o 5. Dos 101 artigos que foram catalogados a partir de tais descritores, 30 artigos foram selecionados, pois atendiam aos critérios de inclusão e exclusão (Figura 1).

Figura 1. Fluxograma de análise dos estudos da revisão

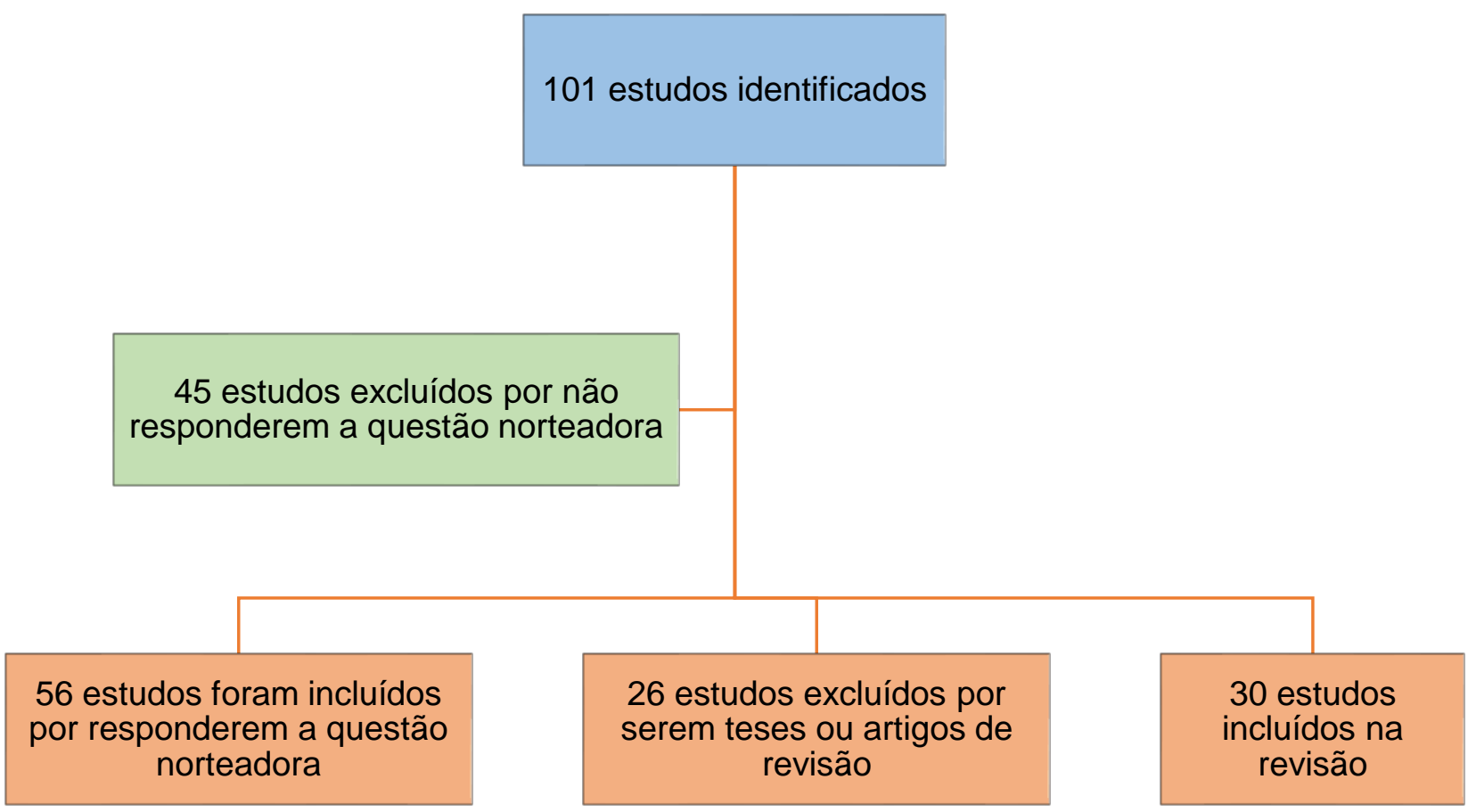

Fonte: elaborada pelos autores. 
Dos 30 artigos selecionados para a quarta etapa da pesquisa, 14 artigos discutiam a judicialização da assistência farmacêutica, perfazendo a categoria de análise 1; 10 artigos abordavam a judicialização do direito à saúde, completando a categoria de análise 2; e seis artigos debatiam sobre a judicialização da saúde pública, preenchendo a categoria de análise 3. Sobre tal organização temática, vale destacar que a distribuição dos artigos se deu tanto pelos descritores utilizados na pesquisa quanto pelo conteúdo de tais publicações. Ou seja, aqueles que abordavam a temática da judicialização em saúde como a busca pelo sistema judiciário como a última alternativa para a obtenção do medicamento ou tratamento ora negado pelo sistema de saúde público ou privado se enquadravam na categoria 1. Já aqueles que condiziam com a categoria 2 se alinharam ao tema da judicialização em saúde como expressão das reivindicações e modos de atuação legítimos de cidadãos e instituições, para a garantia e promoção dos direitos de cidadania amplamente afirmados nas leis. Por fim, os artigos que se adaptavam a categoria 3 anunciavam o tema da judicialização em saúde como modelo adversarial entre a sociedade, que figura como demandante, e o Estado, que é o responsável pelas políticas públicas de saúde (Executivo) ou por resolver conflitos no âmbito da saúde de ordem política, social ou moral (Judiciário).

\section{Resultados e Discussão}

Dentre as 30 publicações encontradas, a maioria concentra-se no período de 2014 a 2017, onde verificamos a publicação de 19 artigos, representando (62\%) dos artigos identificados, com destaque para o ano de 2014, que contabiliza sete publicações, ou seja (37\%) dos artigos do período (Gráfico 1).

Gráfico 1. Quantidade de artigos analisados, divididos por ano de publicação (2008 a 2017)

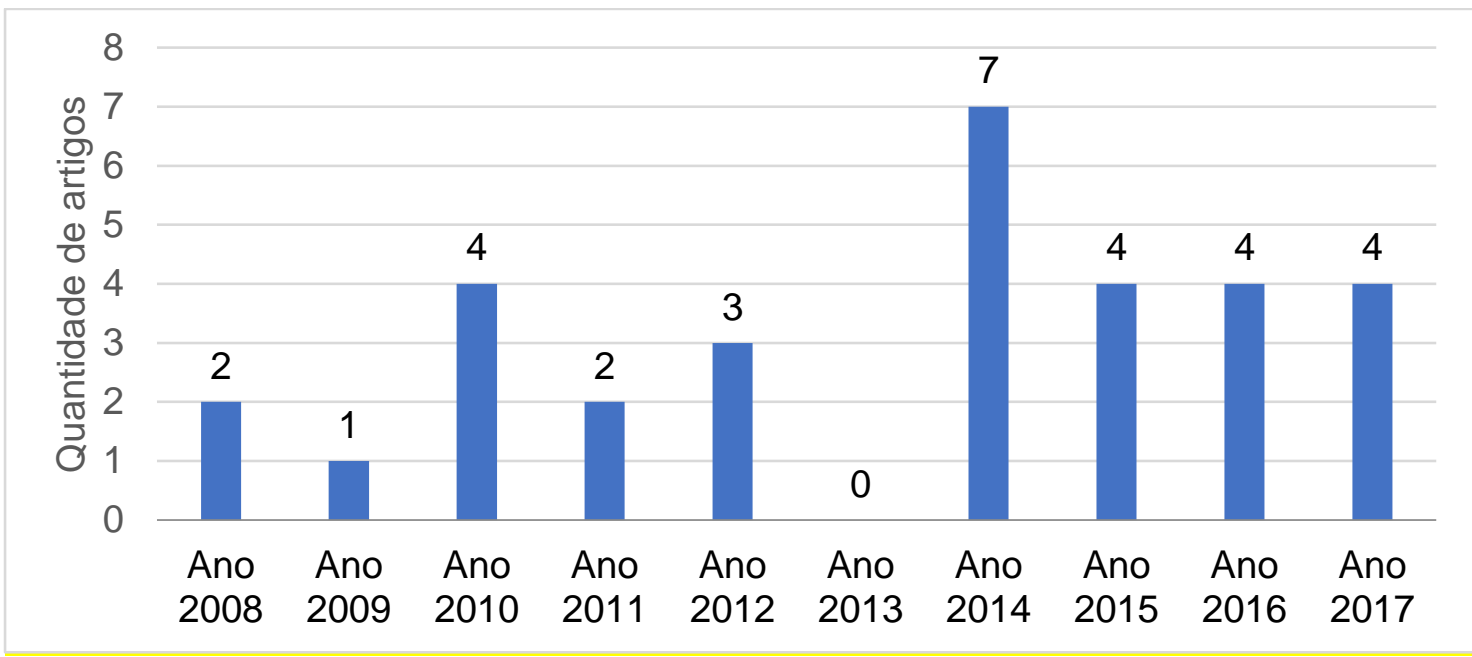

Fonte: elaborado pelos autores. 
Podemos verificar que, dos 30 trabalhos analisados que versam sobre a temática da judicialização da saúde, a grande maioria deles abordam a conceituação do tema sobre três grandes categorias (judicialização e assistência farmacêutica; judicialização e direito à saúde; e judicialização e práticas de gestão), conforme Quadro 1.

Ao se analisar os dados, verificamos o predomínio do tema da judicialização vinculada à assistência farmacêutica, com ênfase no acesso a medicamentos, totalizando cerca de $47 \%$ dos artigos, seguido pelo eixo judicialização do direito à saúde que configura 33\% e, por fim, o eixo da judicialização e práticas de gestão como o menor índice (20\%) dos trabalhos avaliados.

Quadro 1. Artigos organizados por categorias e os possíveis determinantes da judicialização (2008 a 2017)

\begin{tabular}{|c|c|}
\hline $\begin{array}{l}\text { Categorias vinculadas à } \\
\text { judicialização }\end{array}$ & Possíveis determinantes para a judicialização \\
\hline $\begin{array}{l}\text { Judicialização da assistência } \\
\text { farmacêutica (14 artigos - 47\%) }\end{array}$ & $\begin{array}{l}\text { - incentivo à cultura de medicalização; } \\
\text { - preços abusivos de medicamentos; } \\
\text { - falta de estoque de medicamentos; } \\
\text { - falta de padronização do uso; } \\
\text { - medicamentos que não constam na lista de assistência } \\
\text { farmacêutica; } \\
\text { - requerimento de medicamentos em fases ainda } \\
\text { experimentais de pesquisa clínica; } \\
\text { - associação entre médicos e escritórios de advocacia nas } \\
\text { solicitações dos medicamentos; } \\
\text { - vazios assistenciais (na hipótese de doença que não tem } \\
\text { protocolo clínico elaborado pelo SUS); } \\
\text { - conflito entre evidência científica e opinião médica } \\
\text { (quando há prescrição de medicamento fora da bula ou } \\
\text { discordante dos protocolos do SUS } \\
\text { - ineficiência estatal em muitos aspectos da prestação do } \\
\text { serviço à saúde da população. }\end{array}$ \\
\hline $\begin{array}{l}\text { Judicialização do direito à saúde } \\
\text { (10 artigos - 33\%) }\end{array}$ & $\begin{array}{l}\text { - o baixo financiamento, a ausência de parâmetros que } \\
\text { orientem o padrão de integralidade; } \\
\text { - a gestão pública insatisfatória; } \\
\text { - a falta de sentimento de pertencimento da população } \\
\text { com o direito a saúde; } \\
\text { - incapacidade do modelo operacional do SUS; } \\
\text { - falta de políticas públicas de saúde que apoiem ou } \\
\text { coincidam com as reinvindicações do paciente; } \\
\text { - falha da autoridade de saúde pública em cumprir com as } \\
\text { políticas existentes, geralmente devido à falta de uma definição } \\
\text { clara da divisão de autoridade entre entidades federais. }\end{array}$ \\
\hline
\end{tabular}


Judicialização e práticas de gestão (6 artigos - 20\%)
- necessidade de concepção de saúde como direito;

- limitações do SUS real;

- falta de recursos do estado e suplantando o somatório dos ligados à insuficiência da rede de serviços e de médicos;

- falhas encontradas nas políticas públicas;

- deficiências no acesso e cobertura as ações e serviços de saúde no SUS.

- Falta de resolutividade, por parte do SUS, frente as necessidades individuais e coletivas de saúde.

Fonte: elaborado pelos autores.

As três abordagens temáticas trazem leituras diferentes do tema: a primeira categoria entende a judicialização como um novo papel do Poder Judiciário, identificando-o como um fenômeno desafiador e multifacetado, e normalmente faz um recorte da judicialização tratando apenas da vertente de acesso a medicamentos, por ser uma das maiores demandas da judicialização.

Algumas causas são apontadas como fomentadora da judicialização da assistência farmacêutica, principalmente para a solicitação de medicamentos, como por exemplo, a pressão da indústria farmacêutica, a falta de medicamentos disponíveis com regularidade, falta de padronização do uso e registro de medicamento no país, pela cultura da medicalização etc.

Segundo Biehl (15), o sistema público de saúde é visto menos como prevenção e assistência básica, e mais como acesso a medicamentos e assistência terceirizada à comunidade - ou seja, a saúde pública tem sido cada vez mais farmaceuticalizada e privatizada. Percebe-se que o interesse do capital das indústrias farmacêuticas é um elemento que reverbera o entrave da judicialização.

Muitas vezes, os magistrados tomam suas decisões sem a devida análise técnica a respeito da real utilidade e segurança do remédio, uma vez que muitas demandas são de medicamentos que não chegaram nem a serem registrados por órgãos regulatórios nacionais, como a Agência Nacional de Vigilância Sanitária (Anvisa), o que pode trazer, inclusive, danos à saúde do indivíduo. A garantia imediata do direito à saúde por meio de fármacos contorna questões sobre as limitações da política e de recursos, bem como a base de evidências da eficácia de novos medicamentos (16).

Apesar da maioria das demandas judiciais focarem em fármacos de alto custo, outro determinante encontrado diz respeito à judicialização de medicamentos que já são disponibilizados pelo Sistema Único de Saúde (SUS), o que pode ser um indicador de falhas administrativas em alguma etapa da garantia da entrega do remédio. Vieira (17), em sua 
pesquisa, verificou que $62 \%$ dos itens solicitados fazem parte de listas de medicamentos de programas do SUS. Esse novo viés encontrado pode ser sugestivo para a falta de conhecimento do médico acerca dos remédios ofertados por listas oficiais do sistema, como, por exemplo, a Relação Nacional de Medicamentos Essenciais (Rename). Pepe et al (18) ratifica esse pensamento ao afirmar que esse fato aponta para a existência de problemas relacionados à aquisição, distribuição e dispensação de medicamentos pelo setor público. Como possíveis consequências, pode-se haver o comprometimento da Política Nacional de Medicamentos (PNM), a equidade no acesso e o uso racional de medicamentos no Sistema Único de Saúde.

Cabe destacar que deficiências na esfera jurídica também podem ser um fator que corrobora com o agravamento desse evento. De fato, a judicialização da saúde é uma temática que cresceu exponencialmente nos últimos anos e necessita de uma abordagem mais refinada, haja vista seus profundos impactos no orçamento da saúde pública. Para dimensionar as proporções que esse fenômeno atingiu no Poder Judiciário, em abril e maio de 2009 o STF realizou uma audiência pública com a finalidade de averiguar os desafios apresentados pela judicialização da saúde.

Como um dos resultados dessa audiência, consoante Ribeiro (19), surgiu a Recomendação no 31/2010 do Conselho Nacional de Justiça (CNJ), dispondo sobre orientações aos Tribunais brasileiros sobre a melhor forma de julgamento das ações em saúde. Entre suas principais recomendações, pode-se citar: a orientação para que o Judiciário ouça, quando possível, gestores de saúde antes de deferirem o pedido de urgência, possibilitando um maior diálogo entre órgãos; estabeleçam convênios disponibilizando apoio técnico de médicos e farmacêuticos para subsidiarem suas decisões; e que evitem fornecer autorização de medicamentos ainda não padronizados pela Anvisa.

Como se não bastasse, muitos juízes têm o entendimento de que, ao julgarem seus casos positivamente, embora causem um descompasso às políticas de saúde, estão exercendo sua função constitucional de preencher um déficit que é de responsabilidade do Estado, sendo esses processos uma forma de auxiliar o pleno exercício da democratização dos direitos do cidadão. De acordo com Biehl (16), juízes empregam lógicas idiossincráticas e criam seus próprios padrões ao adjudicar casos sobre o direito à saúde.

Wang et al (20) aprofundam ainda mais essa discussão e afirmam que o Judiciário brasileiro também tende a desconsiderar o impacto orçamentário de uma decisão judicial que obriga o sistema de saúde a fornecer um determinando tratamento. Para os juízes, em 
geral, questões relativas ao orçamento público, como a escassez de recursos e a não previsão de gasto, bem como o não pertencimento do medicamento pedido às listas de medicamentos do SUS, não são razões suficientes para se denegar o pedido de um tratamento médico, dado que este encontra respaldo no direito à saúde assegurado pela Constituição Federal.

A segunda categoria, judicialização do direito à saúde, pauta o entendimento da judicialização com base na Constituição Cidadã de 1988, apontando-a como uma estratégia dos sujeitos para garantir o acesso a bens e serviços de saúde. Nesse sentido, direcionando a judicialização para resposta do Estado frente uma solicitação do sujeito de direito. Desde que a Constituição Federal de 1988 passou-se a reconhecer a saúde como direito de todos e dever do Estado, o entendimento social desse direito se reorganizou também juridicamente. Desde então quando as demandas não são atendidas via SUS, passa a ser requerida do Judiciário uma resposta à necessidade de saúde. Alguns fatores podem ter contribuído para o aumento das publicações entre os anos de 2014 a 2017, por exemplo a visibilidade do tema no âmbito nacional e, posteriormente, a instituição de arcabouços jurídicos determinados para tratar inicialmente de atender o grande fluxo das demandas que cresciam constantemente.

Podemos citar a Audiência Pública no 04, realizada entre abril a maio de 2009 pelo STF; e como resultado dessa Audiência a Recomendação n. 31/2010 do CNJ; em 2010 a criação do Fórum Nacional para o monitoramento e resolução das demandas de assistência à saúde, através da Resolução no 107 do CNJ; e da Recomendação de nํ4ㄴ também do CNJ no ano de 2013, que versa sobre a criação de Varas especiais, estabelecendo que os Tribunais de Justiça e aos Tribunais Regionais Federais promovam a especialização de Varas para processar e julgar ações que tenham por objeto o direito à saúde pública e para priorizar o julgamento dos processos relativos à saúde suplementar. Assim, em 2013 a preocupação não versava apenas em atender o grande fluxo de demandas judiciais, mas entendê-las e buscar soluções para o impacto nos orçamentos destinados à política de saúde.

Para Ferraz (21), a judicialização do direito à saúde gera realidades sociomédicas extremamente complexas, além de enormes desafios administrativos e fiscais que, segundo especialistas, têm o potencial de aumentar as desigualdades na prestação de serviços de saúde. 
Em síntese, entende-se que esse fenômeno é uma estratégia cada vez mais usada pelos usuários a fim de garantir o seu direito, quando lesado. Em contrapartida, como os custos com as demandas judiciais não fazem parte do orçamento da saúde, há o surgimento de uma problemática que afeta, sobretudo, a coletividade da população em detrimento de demandas individuais daqueles com maior poder de reivindicação.

Por isso, a judicialização é um processo que influi de certa forma no aporte de recursos para o atendimento de demandas particulares, gerando um desequilíbrio orçamentário responsável por acentuar o enfraquecimento da política de saúde. Essa redução do orçamento, inclusive, pode ser um fator catalisador do próprio fenômeno, uma vez que ocorrerá a hipossuficiência de setores da saúde responsável por causar mais insatisfação do usuário e uma maior probabilidade de recorrência ao Poder Judiciário, a fim de solucionar essa situação.

Os usuários têm recorrido ao Poder Judiciário como solução para o acesso a medicamentos de alto custo, diagnósticos, internações ou procedimentos. Esse ato, conjuntamente com a requisição de procedimentos e medicamentos não fornecidos pelo SUS, é conhecido por todos como o fenômeno da judicialização da saúde (22). Apesar desse evento ser visto de uma boa maneira por boa parte dos juristas, haja vista que é uma forma de garantir a plenitude dos direitos de qualquer cidadão através da exigência do Estado pela prestação do amparo à saúde, a judicialização causa impactos acentuados, sobretudo no âmbito da gestão dos recursos públicos.

Os números também são expressivos quando se compara a evolução desse fenômeno ao longo dos anos no âmbito nacional. O gasto do Ministério da Saúde com medicamentos cuja dispensação foi determinada por ordem judicial passou de $R \$ 2.5$ milhões em 2005 para aproximadamente $R$ \$ 266 milhões em 2011 (23). Com relação a essa situação Carvalho (24), em recente pesquisa inerente ao perfil dos gestores municipais de saúde, identificou que $64 \%$ dos respondentes apontam a necessidade de ampliar o conhecimento dos órgãos de controle sobre a dinâmica da gestão no âmbito do SUS como agenda nacional, com relação à judicialização.

Para Ventura et al (2), a problemática central trazida para o Direito e a Saúde - que se expressa no fenômeno da judicialização da saúde - é a de como o Estado, no âmbito dos Poderes Executivo, Legislativo ou Judiciário, deve proteger as pessoas dos riscos das novidades oferecidas pelo mercado de saúde, que, não raramente, cria necessidades para vender soluções. 
Além disso, foi constatado que nem sempre os profissionais médicos têm o cuidado em prescrever o medicamento mais acessível à comunidade, podendo ser considerado um determinante à medida que o paciente, muitas vezes, não conseguirá acesso ao remédio. Leitão (25), em sua pesquisa, afirma que apesar da falta de acesso aos receituários, durante a análise, foi possível identificar nos autos processuais que não havia o compromisso da maioria dos prescritores em registrar na receita o medicamento pelo nome genérico. Diametralmente oposto a isso, a Lei no $9.787 / 1999$ determina a obrigatoriedade da adoção do nome genérico nas prescrições médicas.

$\mathrm{Na}$ terceira categoria, a judicialização é entendida como a relação que envolve os operadores do direito e aspectos inerentes às práticas de gestão, apontando aspectos amplos e diversificados para a causa da judicialização, passando por processos que para Ramos et al (26) vão desde a necessidade de um maior entendimento sobre a concepção por parte dos usuários sobre o direito à saúde, envolvendo a falta de recursos financeiros, que somados à insuficiência da rede de serviços e de médicos, encontra nas fragilidades da implantação das políticas de saúde, fortes componentes que apontam a limitação do SUS real.

Ramos et al (26), outrossim, apontam que para esses sujeitos, a judicialização facilita o acesso às ações e aos serviços públicos de saúde, mesmo que signifique a resolução de problemas pontuais, não sendo capaz de gerar política ou de aprimorar a política atual, tendendo a individualizar as ações em detrimento do acesso coletivo.

Ainda que a individualização das solicitações por direito à saúde pública não aprimore a política atual, o entendimento das múltiplas determinações das ações judiciais pode apontar caminhos para ampliar e garantir acesso - menos individuais e mais coletivos qualificando assim a política pública.

Embora seja um dos princípios do Sistema Único de Saúde (SUS), o acesso a esse serviço ainda não é feito de forma equânime entre todas as pessoas. As noções de universalidade e equidade estão relacionadas ao princípio da igualdade que, por sua vez, está associado à ideia de justiça no pensamento dos principais filósofos (27). Uma vez não respeitado esse fundamento, a ocorrência de entraves maiores decorrentes desse problema inicial é inerente, como no caso de se recorrer à justiça para a garantia dessa igualdade de acesso não só a procedimentos, como também a outros instrumentos de cuidado ofertados pelo sistema, gerando um déficit no orçamento na prestação dos serviços de saúdes para os outros pacientes. 
Fica claro que a complexidade desse fenômeno ocorre devido às subjetividades que estão inerentes ao próprio processo, independentemente de qual esfera ou fluxo foi utilizado para a garantia do direito universal à saúde resguardando pela nossa Constituição Federal de 1988. Entretanto, ao se deparar com um novo prisma, percebemos a escassez da sistematização de informações sobre quais as causas da judicialização, que chamamos de determinantes da judicialização da saúde.

Em suma, a judicialização da saúde, por um lado, é um acontecimento positivo. Tornou-se mais um instrumento para a garantia da saúde, sendo um direito de todos e dever do Estado, garantido mediante políticas sociais e econômicas que visem à redução do risco de doença e de outros agravos e ao acesso universal e igualitário às ações e serviços para sua promoção, proteção e recuperação (1).

Em contrapartida, esse fenômeno atesta lacunas do Estado em promover uma saúde igualitária para todos, ratificando a insuficiência na garantia plena desse direito, tendo como principal efeito negativo o desequilíbrio na administração dos recursos destinados à saúde, o que afeta o cumprimento, em parte, de ações e serviços de saúde. Constata-se, ainda, que é um evento de extrema complexidade, o qual possui causas multifacetadas e, muitas vezes, enraizadas em ações já solidificadas e familiarizadas no contexto em que vivemos. Deve-se encontrar alternativas que minimizem esse processo, de modo que o Estado garanta a efetivação dos direitos sociais, sem precisar da intervenção do Poder Judiciário, e isso aparece de forma robusta na revisão realizada.

Foi possível também, por meio da leitura dos artigos da revisão de literatura, inferir que o fenômeno da judicialização da saúde não pode ser classificado como algo pejorativo ou oneroso para o Sistema Único de Saúde, visto que se obteve diversas conquistas históricas no âmbito da saúde como: 1) o direito ao tratamento dos usuários portadores do vírus da imunodeficiência humana (HIV); 2) o direito ao tratamento e assistência dos pacientes portadores de doenças raras e raríssimas; 3) procedimentos de alto custo (cirurgias, exames) e 4) medicamentos que ainda não se encontram na lista da Rename.

\section{Conclusão}

A revisão de literatura com os trabalhos que versam sobre a temática da judicialização da saúde nos possibilita afirmar que ainda é baixa a produção científica no que tange a identificação e sistematização de aspectos que tratem dos determinantes da judicialização da saúde no Brasil. Por isso, a produção de estudos e pesquisa que propiciem não só a 
discussão conceitual sobre a temática é importante, mas principalmente a discussão inerente aos processos e práticas vinculados aos determinantes da judicialização na área da saúde, na perspectiva de apontar caminhos para o seu enfrentamento.

Mesmo com as limitações que o estudo apresentou, quanto à quantidade das bases de dados consultadas, foi possível identificar caminhos que permitem uma leitura preliminar sobre a temática e que dizem respeito à busca da articulação de processos e aspectos inerentes à efetiva garantia do direito à saúde, à observação do descumprimento de normas e regras que tem como desfecho a aplicação de uma sanção ao gestor na perspectiva da prestação do serviço ao cidadão e no desconhecimento da dinâmica do SUS.

Outros aspectos a serem destacados dizem respeito à pressão da mídia e da indústria farmacêutica, com a introdução de novas drogas; e tratamentos muitas vezes não regulamentados pelas instituições sanitárias, pela fragilidade do modelo de atenção que tem como uma das consequências os vazios assistências, a dificuldade no acesso a ações e serviços de saúde e a consequente (re)pressão de demanda no componente do financiamento, a qual deixa claro a relação direta entre a falta ou a má aplicação dos recursos e a judicialização.

Sendo assim, existe a necessidade de se ampliar o rol de descritores, incluindo a pesquisa em livros e dissertações, processos que consideramos imprescindíveis no aprimoramento da construção de estudos que nos auxiliem a compreender de forma sistémica as características dos fatores vinculados aos determinantes da judicialização, sendo para tanto imprescindível a realização de novos estudos que abordem essa temática.

\section{Referências}

1. Brasil, Constituição (1988). Constituição da República Federativa do Brasil. Brasília (DF): Senado Federal; 1988

2. Ventura M, Simas L, Pepe VLE, Schramm FR. Judicialização da saúde, acesso à justiça e a efetividade do direito à saúde. Revista de Saúde Coletiva. 2010;20(1):77-100.

3. Pepe VLE, Figueiredo TA, Simas L, Castro-de-Osório CGS, Ventura M. A Judicialização da saúde e os novos desafios da gestão da assistência farmacêutica. Revista Ciência \& Saúde Coletiva. 2010;15(5):2405-2414.

4. Distrito Federal. Consultoria Jurídica da Advocacia Geral da União. Ministério da Saúde. Judicialização da saúde no âmbito da união em números: recursos extraordinários no 566471 e no 657718. Brasília: Ministério da Saúde; 2017 [acesso em 15.mar.2020]. 7 slides, color. Disponível em:

https://portalarquivos2.saude.gov.br/images/pdf/2017/maio/17/JUDICIALIZACAO\%20DA\% 
20SAUDE\%20NO\%20AMBITO\%20DA\%20UNIO\%20EM\%20NUMEROS\%20Recursos\%20 Extraordinrios\%20566471\%20e\%20657718.pdf

5. Gomes D, Souza C, Silva F, Porto J, Morais I, Ramos M, et al. Judicialização da saúde e a audiência pública convocada pelo Supremo Tribunal Federal em 2009: o que mudou de lá para cá? Revista Saúde em Debate. 2014;38(100) 139-156.

6. Marques SB. Judicialização do Direito a Saúde. Revista de Direito Sanitário. 2008; 9(2): 65-72.

7. Brasil. Decreto no 7.508, de 28 de junho de 2011 Regulamenta a Lei no 8.080 , de 19 de setembro de 1990, para dispor sobre a organização do Sistema Único de Saúde - SUS, o planejamento da saúde, a assistência à saúde e a articulação interfederativa, e dá outras providências. Diário Oficial da União. 29 de jun 2011.

8. Fleury S. Judicialização pode salvar o SUS. Revista Saúde em Debate. 2012;36(93):151-162.

9. Azabe PHM. Dimensão Jurídica das Políticas Públicas. In: Bucci MPD. (Org) Políticas Públicas: reflexões sobre o conceito jurídico. São Paulo: Saraiva; 2006. p.51-74.

10. Bucci MPD. O conceito de políticas públicas em direito. In Políticas Públicas: reflexões sobre o conceito jurídico. São Paulo: Saraiva; 2006. p.1-40.

11. Marques SB, Dallari SG. A garantia do direito a assistência farmacêutica no Estado de São Paulo. Revista de Saúde Pública. 2007;41(2):101-107.

12. Chieffi AL, Barata RB. Judicialização da política pública de assistência farmacêutica e equidade. Caderno de Saúde Pública. 2009;25(8):1839-1849.

13. Mendes KDS, Silveira RCCP, Galvão CM. Revisão integrativa: método de pesquisa para a incorporação de evidências na saúde e na enfermagem. Texto Contexto Enfermagem. 2008;17(4):758-764.

14. Carvalho MCM de. Metodologia Científica, Fundamentos e Técnicas: Construir o saber. 4. ed. São Paulo: Papirus; 1994.

15. Biehl J. Will to live: Aids therapies and the politics of survival. Princeton: Princeton University Press; 2007.

16. Biehl J, Petryna A. Tratamentos jurídicos: os mercados terapêuticos e a judicialização do direito à saúde. Revista de História/Ciências/Saúde Manguinhos. 2016;23(1):173-192.

17. Vieira FS, Zucchi P. Distorções causadas pelas ações judiciais à política de medicamentos no Brasil. Revista de Saúde Pública. 2007;41(2):214-222.

18. Pepe VLE. Caracterização de demandas judiciais de fornecimento de medicamentos "essenciais" no Estado do Rio de Janeiro, Brasil. Caderno de Saúde Pública. 2010;26(3):461-471. 
19. Ribeiro, DS. O direito à saúde em tempos neoliberais: a judicialização da saúde como estratégia para a garantia de direitos? [Dissertação]. Juiz de Fora: Universidade Federal de Juiz de Fora; 2014.

20. Wang DWL, Vasconcelos NP, Oliveira VE, Terrazas FV. Os impactos da judicialização da saúde no município de São Paulo: gasto público e organização federativa. Revista de Administração Pública. 2014; 48(5): 1191-1206.

21. Ferraz OLM. The right to health in the courts of Brazil: worsening health inequities? Health and Human Rights: An International Journal. 2009;11(2):33-45.

22. Chagas CP, Santos FP. Efeitos do gasto com a judicialização da saúde no orçamento da Secretaria Estadual de Saúde do Distrito Federal entre 2013 e 2017. Revista Cadernos Ibero-Americanos de Direito Sanitário. 2018 abr./jun; 7(2):47-172.

23. Silva GBJ, Dias ER. Avaliação da satisfação dos usuários de um serviço de saúde público-privado no nordeste do Brasil e a Judicialização da saúde. Revista de Direito Sanitário. 2015;17(2):13-29.

24. Carvalho ALB, Ouverney.ALM, Carvalho MGO, Machado NMS. Enfermeiros (as) gestores (as) no Sistema Único de Saúde: perfil e perspectivas com ênfase no Ciclo de Gestão 2017-2020. Ciência \& Saúde Coletiva. 2020;25(1):211-222.

25. Leitão LCA, Silva PCD, Simões MCS, Simões AEO, Alves BC, Barbosa IC, Pinto MEB. Análise das demandas judiciais para aquisição de medicamentos no estado da Paraíba. Revista de Saúde Social. 2016;25(3):800-807.

26. Ramos RS, Gomes AMT, Guimarães RG, Santos El. A Judicialização da saúde contextualizada na dimensão prática das respresentações sociais dos profissionais de saúde. Revista de Direito Sanitário. 2017; 8(2):18-38.

27. Perelman C. Ética e direito. São Paulo: Editora Martins Fontes; 1996.

\section{Colaboradores}

Carvalho ALB e Santos OAN contribuíram com a concepção e desenho do artigo; redação do artigo, revisão crítica do seu conteúdo e aprovação da versão final. Diniz AMS e Nascimento RV contribuíram com a concepção do artigo e análise e interpretação de dados. Batista BNM e Barbosa DS contribuíram com a redação do artigo e revisão crítica de seu conteúdo. Andrade Silva ERR contribuiu com a análise e interpretação de dados, redação do artigo e revisão crítica de seu conteúdo. 
Como citar este artigo:

Carvalho ALB, Diniz AMS, Batista BNM, Barbosa DS, Andrade Silva ERR, Santos OAN, Nascimento RV.

Determinantes da judicialização da saúde: uma análise bibliográfica. Cadernos Ibero-Americanos de Direito Sanitário. 2020 out./dez.; 9(4): 117-134.

https://doi.org/10.17566/ciads.v9i4.670 